\title{
EVALUATION OF SQUEEZE EFFECT IN A GAS THRUST BEARING
}

\author{
FEDERICO COLOMBO, MONA MORADI, TERENZIANO RAPARELLI, ANDREA TRIVELLA \& \\ VLADIMIR VIKTOROV \\ Politecnico di Torino, Italy
}

\begin{abstract}
The squeeze effect of a gas thrust bearing is calculated using both a distributed parameters (DP) model and a lumped parameters (LP) model. A rectangular thrust bearing with no supply holes is considered in the analysis. In dynamic conditions, pressure values $\mathrm{p}_{1}$ and $\mathrm{p}_{2}$ are calculated. In steady-state conditions, these values coincide with ambient pressure $\mathrm{p}_{\mathrm{a}}$. The developed LP model was validated with the DP model for a pad shape ratio (length/width) in the range of 0.5 to 2 . Both models are validated in the literature in about a 1D parallel slider. The LP model has the advantage of faster implementation in comparison to the DP model. The LP model is also linearized by using a perturbation method to obtain the analytic expression of the dynamic stiffness. Therefore, it is possible to explain the effect of geometrical parameters on stiffness and damping coefficients.

Keywords: squeeze film air thrust bearing, lumped parameters model, dynamic stiffness.
\end{abstract}

\section{INTRODUCTION}

Recently in literature, most of the air bearings are analyzed with distributed parameters (DP) methods, using finite difference technique (FDM) [1], [2] or finite element method (FEM) [3], [4]. Also, CFD models can be used to study the pressure distribution; for example, in [5] the pressure depression effect in aerostatic thrust bearing is investigated. In [6], [7] CFD and FDM methods are applied to investigate static and dynamic behavior of bearings with small supply holes, the numerical results are also experimentally verified. In [8] a multi-physics finite element model is adopted to consider the interaction between the air flow dynamics and the structural flexibility of the bearing.

Generally, all these methods achieve high precision in results but the solution can require long computation time due to the high number of nodes used for the discretization of the pad surface. Also, the identification of the parameters that influence the behavior is not easy.

A lumped parameters model can be alternatively used as a fast design tool for air bearings. It does not require the use of commercial software and can be successfully adopted both for static and dynamic study. Due to its quite good precision and low number of parameters, this method allows an easy design and understanding of the phenomena.

For simple geometries, analytical and lumped parameters methods were preferred in the past, when the computational speed was not high. Paper [9] presents analytical study of static characteristics of the bearings, papers [10], [11] show dynamic performance and stability criteria of aerostatic bearings adopting linearized equations of motion.

Recently in [12] a lumped parameters model is proposed to study the static performance of a multiple supply holes rectangular pad. The results of load capacity, stiffness and air consumption were obtained in dimensionless form by chancing position, numbers and diameters of supply holes.

About the dynamic behavior of air pads in [13] the time response of the force to a step variation of the air gap is analyzed. Results with lumped and distributed model are in good agreement. 
Further studies on the dynamic of air pads are currently focused on their dynamic stiffness and damping. By applying a sinusoidal variation of the air-gap it is possible to determine the stiffness and the damping of the pad for different frequency of the input signal.

In particular this paper analyzes theoretically the squeeze film phenomenon of a rectangular pad in absence of the supply inlet holes. The dynamic lumped model is presented; it considers the flow rate due only to the variation of the air gap. The flow rate is linearized to obtain the transfer function of the pressure force respect to the air gap; the dynamic stiffness and damping are calculated at different frequencies. The numerical results are compared with those of a distributed model and are also validated with literature in case of a 1D parallel slider [14].

\section{THE LP MODEL}

\subsection{Air bearing geometry}

A squeeze film rectangular air bearing of sides $L_{\mathrm{x}}$ and $L_{\mathrm{y}}$ with uniform air gap is considered. The pad shape ratio is:

$$
\beta=\frac{L_{y}}{L_{x}}
$$

In steady-state conditions the pressure under the pad is equal to ambient pressure $p_{\mathrm{a}}$; on the contrary in case the air gap changes with time the pressure is not uniform due to squeeze effect. In the developed LP model two pressure values are considered: pressure $p_{2}$ is supposed to be constant at distance $l$ from the borders and pressure $p_{1}$ is supposed to be constant inside volume $V_{1}$, which is an inner rectangle at distance $2 l$ from the borders. Fig. 1 represents the pad with the supposed pressure distribution. In volume $V_{2}$, defined outside volume $V_{1}$, the pressure is supposed linear from the perimeter in which $p=p_{2}$. Inside volume $V_{1}$ the pressure is supposed constant and equal to $p_{1}$.
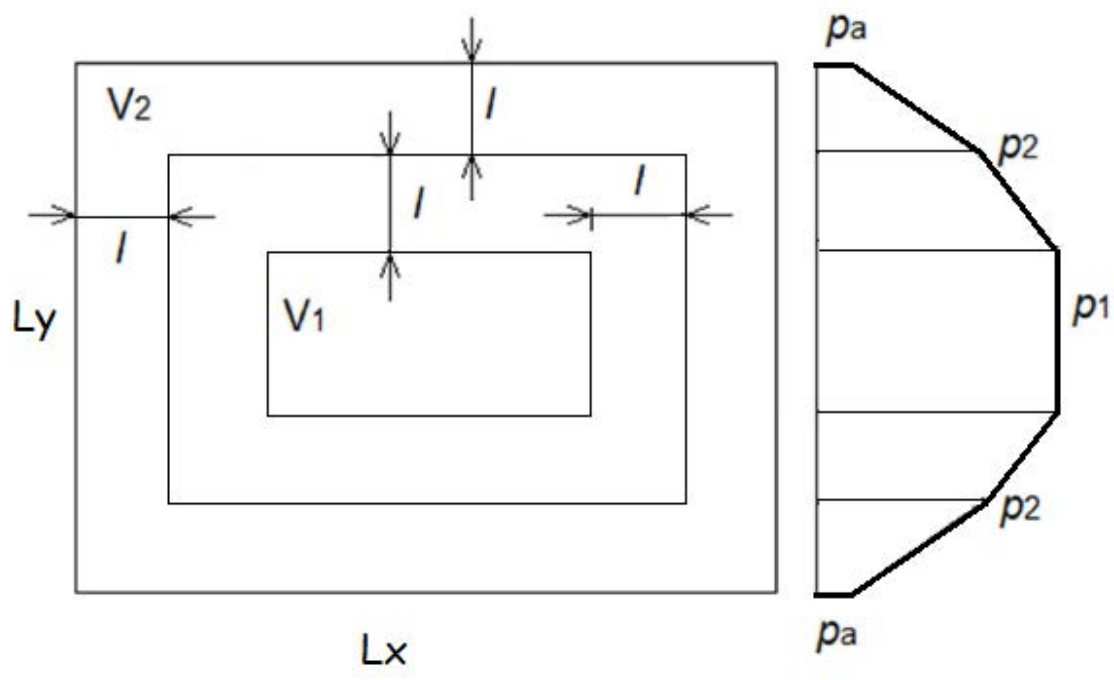

Figure 1: Sketch of the pad and pressure distribution. 


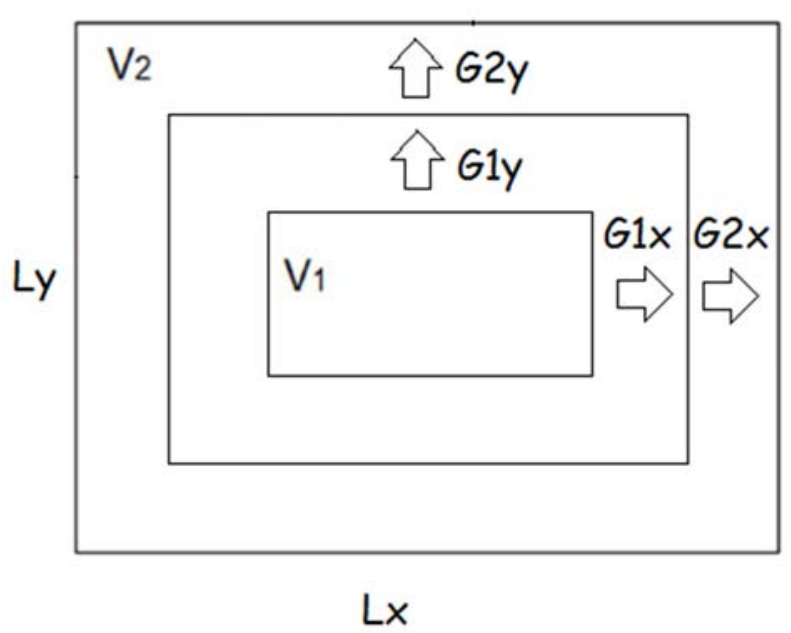

Figure 2: Sketch of the pad with indication of the mass air flows along $\mathrm{x}$ and $\mathrm{y}$ directions.

\subsection{Flow balance}

As illustrated in Fig. 2, mass air flow $G_{1}$ is defined from volume $V_{1}$ to volume $V_{2}$, while air flow $G_{2}$ is defined from $V_{2}$ to the exhaust. These mass flows can be calculated considering the formula for rectangular channels.

$$
\begin{gathered}
G_{1}=G_{1 x}+G_{1 y}=\frac{h^{3}}{6 \mu R T}\left(\frac{L_{y}-2 l}{L_{x}-2 l}+\frac{L_{x}-2 l}{L_{y}-2 l}\right)\left(p_{1}^{2}-p_{2}^{2}\right) \\
G_{2}=G_{2 x}+G_{2 y}=\frac{h^{3}}{12 \mu R T}\left(\frac{L_{y}-2 l}{l}+\frac{L_{x}-2 l}{l}\right)\left(p_{2}^{2}-p_{a}^{2}\right)
\end{gathered}
$$

The mass balance equations for volumes $V_{1}$ and $V_{2}$ are given in eqns (4) and (5):

$$
\begin{gathered}
G_{1}-G_{2}=\dot{m}_{2} \\
-G_{1}=\dot{m}_{1}
\end{gathered}
$$

The air masses $m_{1}$ and $m_{2}$ in volumes $V_{1}$ and $V_{2}$ can be obtained integrating the air density over these volumes under the hypothesis of isothermal expansion:

$$
\begin{aligned}
m_{1} & =\int_{V 1} \frac{p}{R T} d V=\frac{h p_{1}}{R T}\left(\frac{L_{x}-2 l}{2}\right)\left(\frac{L_{y}-2 l}{2}\right) \\
m_{2}=\int_{V 2} \frac{p}{R T} d V & =\frac{h}{R T}\left(p_{2}\left[l\left(L_{x}+L_{y}-4 l\right)+l^{2}+\frac{7}{16}\left(L_{x}-2 l\right)\left(L_{y}-2 l\right)\right]\right. \\
+ & \left.\frac{5 p_{1}\left(L_{x}-2 l\right)\left(L_{y}-2 l\right)}{16}+l p_{a}\left[L_{x}+L_{y}-l\right]\right)
\end{aligned}
$$

At this level, the resulting continuity equation is non-linear. In the next paragraph, we consider the linearized equation. 


\subsection{Linearization of the equations}

Linearization of the problem gives the possibility of obtaining the transfer function between air gap and load capacity so that it is possible to study the frequency response of the pad and analyze the sensitivity of the pad characteristics to the geometrical parameters.

The problem is linearized around the operating point defined by the air gap $\mathrm{h}_{0}$. Using the perturbation method to linearize the problem, all parameters in equations are considered as the sum of a constant steady state term and a dynamic term:

$h=h_{0}+\Delta h(8)$

$p_{1}=p_{0}+\Delta p_{1}(9)$

$p_{2}=p_{0}+\Delta p_{2}(10)$

The steady state values of pressures for a dynamic squeeze film pad are equal to ambient pressure: $p_{0}=p_{a}$.

The continuity equations assume the expressions of eqns (11) and (12)

$$
\begin{gathered}
-G_{1,0}-\Delta G_{1}=\dot{m}_{1} \\
G_{1,0}+\Delta G_{1}-G_{2,0}-\Delta G_{2}=\dot{m}_{2}
\end{gathered}
$$

The steady state air flows are null so the continuity equations simplify into the following

$$
\begin{gathered}
-\Delta G_{1}=m_{1} \\
\Delta G_{1}-\Delta G_{2}=\dot{m}_{2}
\end{gathered}
$$

All parameters are linearized with the first order Taylor expansion:

where

$$
\begin{gathered}
\Delta G_{1}=a_{6} \Delta p_{1}+a_{7} \Delta p_{2}+a_{8} \Delta h=\left[\begin{array}{lll}
a_{6} & a_{7} & 0
\end{array}\right]\left[\begin{array}{c}
\Delta p_{1} \\
\Delta p_{2} \\
\Delta h
\end{array}\right] \\
\Delta G_{2}=a_{9} \Delta p_{2}+a_{10} \Delta h=\left[\begin{array}{lll}
0 & a_{9} & a_{10}
\end{array}\right]\left[\begin{array}{c}
\Delta p_{1} \\
\Delta p_{2} \\
\Delta h
\end{array}\right]
\end{gathered}
$$

$$
\begin{aligned}
a_{6} & =\frac{-h_{0}^{3} p_{0}}{3 \mu R T}\left(\frac{L_{y}-2 l}{L_{x}-2 l}+\frac{L_{x}-2 l}{L_{y}-2 l}\right) \\
a_{7}=-a_{6} & \\
a_{9} & =\frac{h_{0}^{3} p_{0}}{6 \mu R T}\left(\frac{L_{y}-2 l}{l}+\frac{L_{x}-2 l}{l}\right) \\
a_{10} & =\frac{h_{0}^{2}\left(p_{0}^{2}-p_{a}^{2}\right)}{4 \mu R T}\left(\frac{L_{y}-2 l}{l}+\frac{L_{x}-2 l}{l}\right)
\end{aligned}
$$

In the Laplace domain, the time variation of the masses will be

$$
\begin{gathered}
\dot{m}_{1}=a_{1} \Delta \dot{p}_{1}+a_{2} \Delta \dot{h}=\left[\begin{array}{lll}
a_{1} s & 0 & a_{2} s
\end{array}\right]\left[\begin{array}{c}
\Delta p_{1} \\
\Delta p_{2} \\
\Delta h
\end{array}\right] \\
\dot{m}_{2}=a_{3} \Delta \dot{p}_{1}+a_{4} \Delta \dot{p}_{2}+a_{5} \Delta \dot{h}=\left[\begin{array}{lll}
a_{3} s & a_{4} s & a_{5} s
\end{array}\right]\left[\begin{array}{c}
\Delta p_{1} \\
\Delta p_{2} \\
\Delta h
\end{array}\right],
\end{gathered}
$$

where 


$$
\begin{gathered}
a_{1}=\frac{L_{y}-2 l}{2} \frac{L_{x}-2 l}{2 R T} h_{0} \\
a_{2}=\frac{L_{y}-2 l}{2} \frac{L_{x}-2 l}{2 R T} p_{a} \\
a_{3}=h_{0} \frac{5}{16 R T}\left(L_{x}-2 l\right)\left(L_{y}-2 l\right) \\
a_{4}=h_{0} \frac{1}{R T}\left[l\left(L_{x}+L_{y}-4 l\right)+l^{2}+\frac{7}{16}\left(L_{x}-2 l\right)\left(L_{y}-2 l\right)\right] \\
a_{5}=\frac{p_{a}}{R T}\left[2 l\left(L_{x}+L_{y}\right)-4 l^{2}+\frac{3}{4}\left(L_{x}-2 l\right)\left(L_{y}-2 l\right)\right]
\end{gathered}
$$

Finally, the mass balance equations in Laplace domain are written in the compact system:

$$
\left[\begin{array}{cr}
a_{1} s-a_{6} & -a_{7} \\
a_{3} s+a_{6} & a_{4} s+a_{7}+a_{9}
\end{array}\right]\left[\begin{array}{l}
\Delta p_{1} \\
\Delta p_{2}
\end{array}\right]=-\left[\begin{array}{c}
a_{2} s \\
a_{5} s+a_{10}
\end{array}\right] \Delta h
$$

from which it is possible to obtain the pressures $p_{1}$ and $p_{2}$ :

$$
\left[\begin{array}{c}
\frac{\Delta p_{1}}{\Delta h} \\
\frac{\Delta p_{2}}{\Delta h}
\end{array}\right]=-\frac{1}{a_{11}}\left[\begin{array}{c}
a_{7} a_{9} s^{2}+\left[a_{2} a_{10}+a_{7}\left(a_{2}+a_{4}\right)\right] s+a_{2} a_{5} \\
\left(-a_{8} a_{7}+a_{6} a_{10}\right) s^{2}+\left[-a_{1} a_{7}+a_{6} a_{5}-a_{1} a_{10}\right] s-a_{1} a_{5}
\end{array}\right],
$$

where

$$
a_{11}=a_{6} a_{9} s^{2}+\left[-a_{1} a_{9}+a_{6}\left(a_{2}+a_{4}\right)+a_{2} a_{8}\right] s-a_{1} a_{4}
$$

\subsection{Transfer function}

The pad load capacity $F$ is calculated by integration of the pressure distribution under the pad. An expression of the transfer function in Laplace domain is then obtained:

$$
\frac{\Delta F(s)}{\Delta h(s)}=M \frac{\Delta p_{1}(s)}{\Delta h(s)}+N \frac{\Delta p_{2}(s)}{\Delta h(s)},
$$

where $M$ and $N$ are coefficients depending on the geometry of the pad:

$$
\begin{gathered}
M=9 \frac{\left(L_{x}-2 l\right)\left(L_{y}-2 l\right)}{16} \\
N=l\left(L_{x}+L_{y}-2 l\right)+\frac{7}{16}\left(L_{x}-2 l\right)\left(L_{y}-2 l\right)
\end{gathered}
$$

The coefficients of stiffness and damping of the pad can be obtained from the real and imaginary parts of the transfer function:

which has this form:

$$
\frac{\Delta F(s)}{\Delta h(s)}=k(s)+c(s) s
$$

where

$$
\frac{\Delta F(s)}{\Delta h(s)}=\frac{b_{1} s^{2}+b_{2} s+b_{3}}{b_{4} s^{2}+b_{5} s+b_{6}}
$$

$$
\begin{gathered}
b_{1}=-M a_{2} a_{4}-N\left(-a_{3} a_{2}+a_{1} a_{5}\right) \\
b_{2}=-M\left[-a_{6} a_{5}+a_{2}\left(-a_{6}+a_{9}\right)\right]-N\left[-a_{6} a_{2}+a_{1} a_{10}-a_{6} a_{5}\right] \\
b_{3}=(M+N) a_{6} a_{10} \\
b_{4}=a_{1} a_{4}
\end{gathered}
$$




$$
\begin{gathered}
b_{5}=\left[-a_{6} a_{4}+a_{1}\left(a_{7}+a_{9}\right)+a_{7} a_{3}\right] \\
b_{6}=-a_{6} a_{9}
\end{gathered}
$$

If the frequency $\omega$ is introduced it is possible to obtain analytical expressions for stiffness and damping:

$$
\begin{aligned}
\frac{\Delta F(\omega)}{\Delta h(\omega)} & =\frac{\left(b_{3}-b_{1} \omega^{2}\right)\left(b_{6}-b_{4} \omega^{2}\right)+b_{2} b_{5} \omega^{2}}{\left(b_{6}-b_{4} \omega^{2}\right)^{2}+b_{5}^{2} \omega^{2}} \\
& +j \omega \frac{b_{2}\left(b_{6}-b_{4} \omega^{2}\right)-b_{5}\left(b_{3}-b_{1} \omega^{2}\right)}{\left(b_{6}-b_{4} \omega^{2}\right)^{2}+b_{5}^{2} \omega^{2}}
\end{aligned}
$$

\section{COMMENTS AND RESULTS}

\subsection{Static condition}

In static condition stiffness coefficient is:

$$
k(\omega=0)=\frac{b_{3}}{b_{6}}=-\frac{(M+N) a_{10}}{a_{9}}=-(M+N) \frac{3\left(p_{0}^{2}-p_{a}^{2}\right)}{2 h_{0} p_{0}}=0
$$

as $p_{0}=p_{\text {a. }}$. The static damping is

$$
c(\omega=0)=\frac{b_{2} b_{6}-b_{5} b_{3}}{b_{6}{ }^{2}}
$$

In this case damping is not zero. This can be explained due to viscosity which creates a shear stress which opposes to the pad vertical movement.

\subsection{Comparison with the distributed model}

The pad was simulated in dynamic conditions with a distributed parameters software which integrates Reynolds equation under the pad. The air gap was perturbed near $h_{0}=10 \mu \mathrm{m}$ with a sinusoidal function of amplitude $\Delta h=0.5 \mu \mathrm{m}$ at different frequencies:

$$
h=h_{0}+\Delta h \sin (\omega t)
$$

Fig. 3 shows the load capacity $F$ and air gap $h$ vs angle $\omega t$.
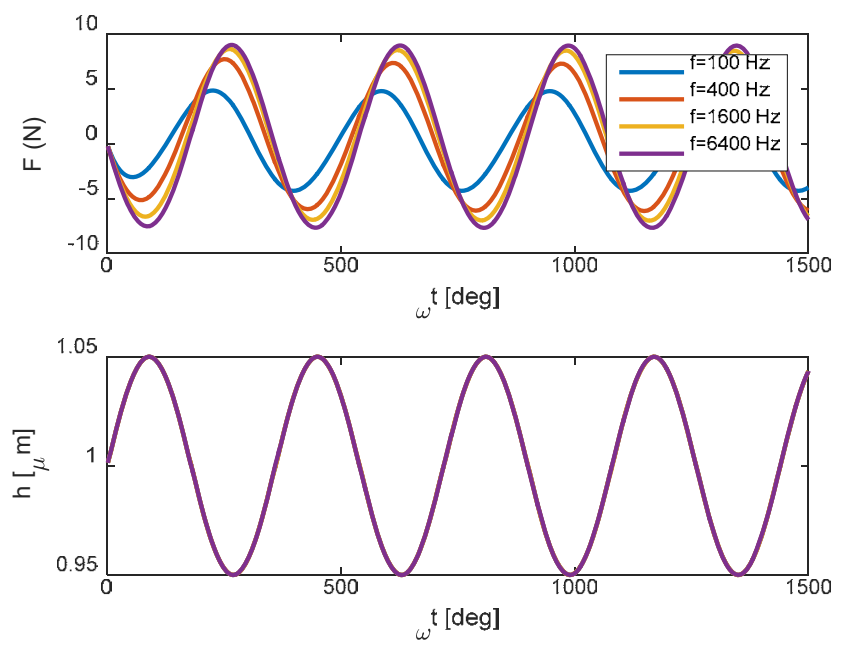

Figure 3: Load capacity and air gap vs angle $\omega t$ for different frequencies. 
From these results coefficients of stiffness and damping were calculated measuring the phase shift between $F$ and $h$. The results are compared in Figs. 4 and 5 with that of lumped model for a pad of size $30 \times 60 \mathrm{~mm}$. Distance $l=5 \mathrm{~mm}$ was assumed to define volumes $V_{1}$ and $V_{2}$.

If compared with the DP model, the LP model is much less expensive in terms of computational time, as it needs few seconds compared to some hours, needed for DP model.

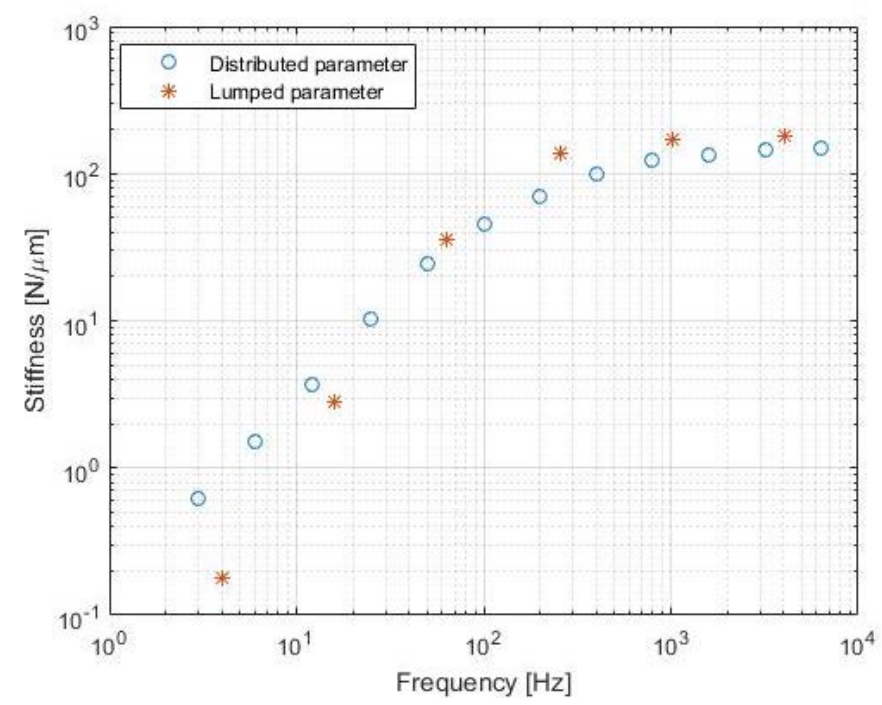

Figure 4: Comparison of the damping obtained with lumped and distributed parameter models.

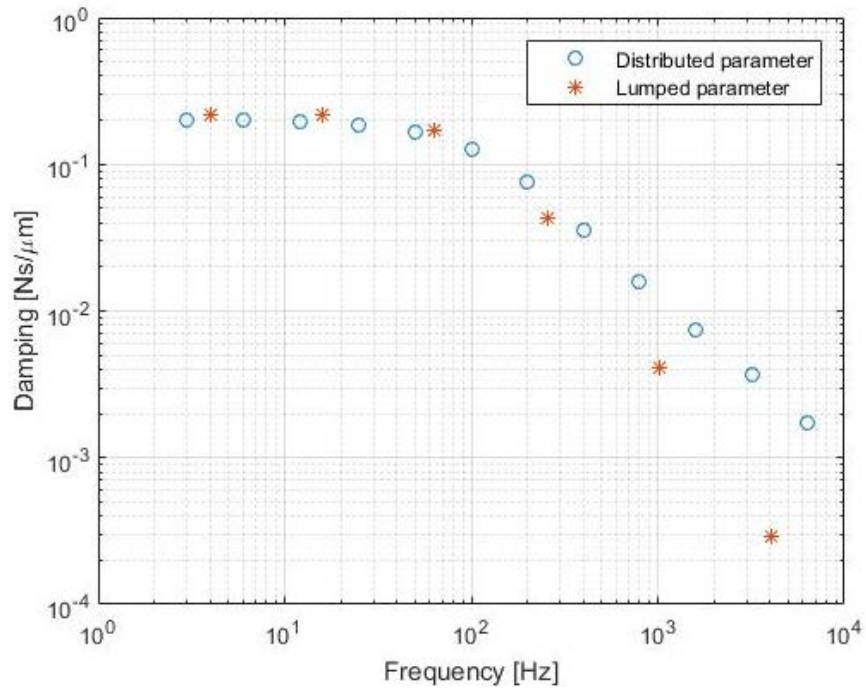

Figure 5: Comparison of the damping obtained with lumped and distributed parameter models. 


\subsection{Literature validation}

The LP model was compared with literature paper [14] in which a 1D slider is considered and an analytical formulation was given. To approach the pressure distribution of a monodimensional slider, a pad with aspect ratio $\beta=5$ was considered. In this case the length of pad side is sufficiently greater than the other side and it is possible to have a good approximation of the mono-dimensional slider.

Stiffness and damping are shown in dimensionless form using the following definition:

$$
\begin{gathered}
\bar{k}=\frac{k h_{0}}{L_{x} L_{y} p_{a}} \\
\bar{c}=\frac{c h_{0} \omega}{L_{x} L_{y} p_{a}}
\end{gathered}
$$

Dimensionless frequency is defined by the squeeze number:

$$
\text { squeeze number }=\frac{24 \mu \pi \omega L_{x}{ }^{2}}{P_{a} h_{0}{ }^{2}}
$$

From the figure, it is evident that the pad of aspect ratio $\beta=5$ has coefficients more similar to that of the mono-dimensional air slider. Moreover, the LP model gives a reasonable result in comparison with literature.

\section{CONCLUSION}

The LP model can be used to obtain the stiffness and damping coefficients of a rectangular squeeze film pad. The results were compared with a distributed parameters model and give good approximation. The LP model was also validated with literature in case of a monodimensional slider.

It is evident that the computational time of LP model is much less than that of distributed model (few seconds instead of many hours), so LP model is a good instrument for design.

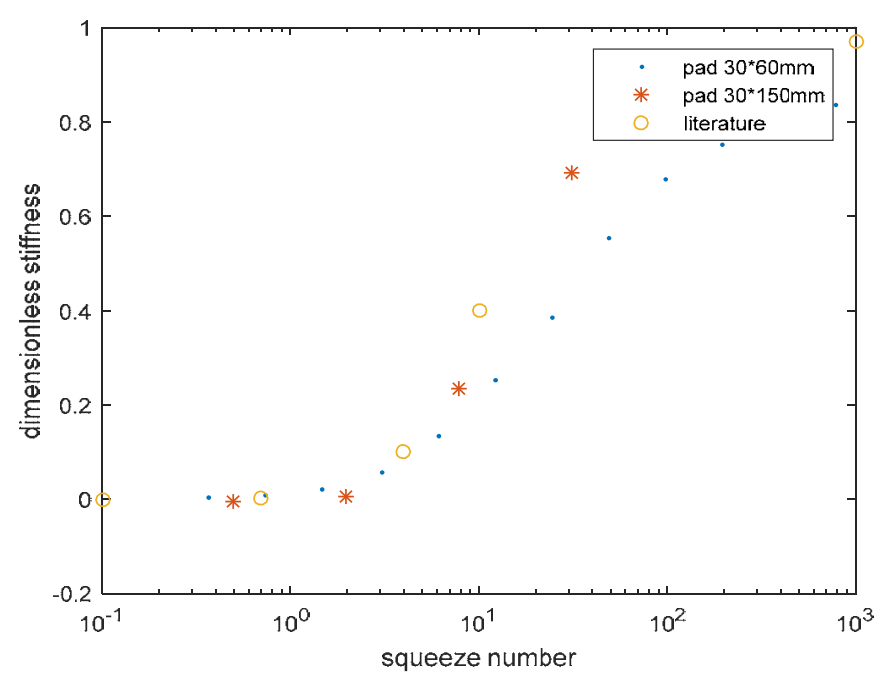

Figure 6: Dimensionless stiffness; comparison between LP model and 1D slider from paper [14]. 


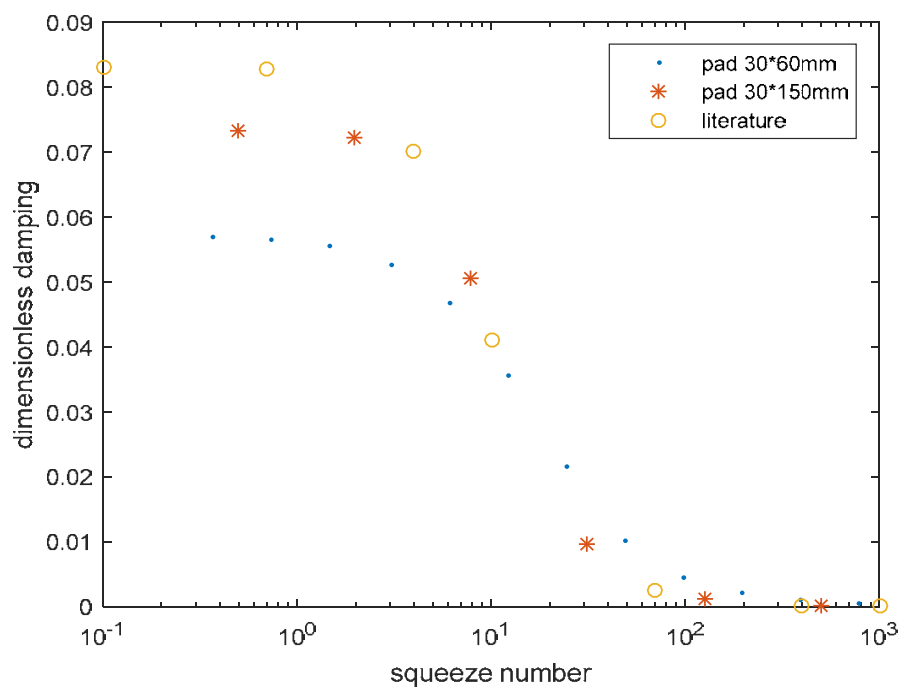

Figure 7: Dimensionless damping; comparison between LP model and 1D slider from paper [14].

\section{REFERENCES}

[1] Stout, K.J., El-Ashkar, S., Ghasi, V. \& Tawfik, M., Theoretical analysis of two configurations of aerostatic flat pad bearings using pocketed orifice restrictors. Tribology International, 26, pp. 265-273, 1993.

[2] Zhang, H., Zhu, C. \& Yang, Q., Approximate numerical solution of hydrodynamic gas journal bearings, ICIRA, Part II, LNAI 5315, pp. 260-268, Springer-Verlag: Berlin Heidelberg, 2008.

[3] Fourka, M., Tian, Y. \& Bonis, M., Prediction of the stability of air thrust bearings by numerical, analytical and experimental methods. Wear, 198, pp. 1-6, 1996.

[4] Li, Y.T. \& Ding, H., Design analysis and experimental study of aerostatic linear guideways used in a high acceleration and high precision xy stage. Proc. IMechE, Part J, Engineering Tribology, 221, 2007.

[5] Eleshaky. M.E., CFD investigation of pressure depression in aerostatic circular thrust bearings. Tribology International, 42, pp. 1108-1117, 2009.

[6] Masaaki, M. \& Yoshimoto, S., Numerical investigation of static and dynamic characteristics of aerostatic thrust bearings with small feed holes. Tribology International, 43, pp. 1353-1359, 2010.

[7] Nishio, U., Somaya, K. \& Yoshimoto, S., Numerical calculation and experimental verification of static and dynamic characteristics of aerostatic thrust bearings with small feedholes. Tribology International, 44, pp. 1790-1795, 2011.

[8] Aguirre, G., Al-Bender, F. \& Brussel, H.V. A multiphysics model for optimizing the design of active aerostatic thrust bearings. Precision Engineering, 34, pp. 507-515, 2010.

[9] Mori, H. \& Miyamatsu, Y., Theoretical flow-models for externally pressurized gas bearings. ASME Journal of lubrication technology, pp. 181-193, 1969.

[10] Licht, L. \& Elrod, H., A study of the stability of externally pressurized gas bearings. J. Appl. Mech., 27(2), pp. 250-259, 1960. 
[11] Bassani, R., Ciulli, E. \& Forte, P., Pneumatic stability of the integral aerostatic bearing: comparison with other types of bearing. Tribology International, vol. 22 (6), (1989), pp. 363-374.

[12] Colombo, F., Raparelli, T., Trivella, A. \& Viktorov, V., Lumped parameters models of rectangular pneumatic pads: static analysis. In: PRECISION ENGINEERING, vol. 42, pp. 283-293, 2015. ISSN 0141-6359

[13] Belforte, G., Colombo, F., Raparelli, T., Trivella, A. \& Viktorov, V., Study of the static and dynamic performance of rectangular air pads by means of lumped parameters models. Proceedings of the 11th Biennial conference on Engineering Systems Design and Analysis ESDA 2012, July 2-4, Nantes, France.

[14] Arghir, M. \& Matta, P., Compressibility effects on the dynamic characteristics of gas lubricated mechanical components, (2009), C.R. Mecanique 337, pp. 739-747. 